\title{
Esophageal atypical carcinoid tumor with tracheal invasion
}

\author{
Ke-Cheng Chen, MD, ${ }^{a}$ Yih-Leong Chang, MD, ${ }^{b}$ Chien-Te Pan, MD, and \\ Yung-Chie Lee, MD, PhD, ${ }^{\text {a }}$ Taipei, Taiwan
}

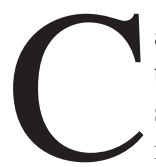

arcinoid tumors of the gastrointestinal tract are distinctive endocrine neoplasms commonly found in the small bowel and appendix; however, they are exceedingly uncommon in the esophagus. It was first reported in 1974 that esophageal carcinoids arise from the argyrophil endocrine cells, which are amine precursor uptake and decarboxylation cells of the mucosa. ${ }^{1}$ The aggressiveness of atypical carcinoid of the esophagus often precludes the use of limited surgical resection and requires adjuvant chemoradiotherapy. ${ }^{2}$ Here we report our successful palliative management of an advanced esophageal atypical carcinoid with tracheal invasion using a covered Ultraflex expandable metallic tracheal stent (Boston Scientific, Galway, Ireland) and oral thalidomide.

\section{Clinical Summary}

A 73-year-old man presented with a 6-month history of progressive dysphagia and 2-kg body weight loss. At the time of presentation, full diet was still tolerable but with a slow pace. No clinical features of carcinoid syndrome were noted. The esophagoscopic examination revealed a $1.7-\mathrm{cm}$ polypoid mass at the upper-third esophagus, $20 \mathrm{~cm}$ below the incisor. Chest computed tomography revealed an esophageal tumor at the upper-third portion with invasion to the trachea (Figure 1,A). The bronchoscope showed multiple intraluminal tumors over the upper trachea, causing mild obstruction. The panendoscopic and bronchoscopic biopsies did not provide an adequate specimen for pathologic examination. Further intervention was suggested to establish a definite diagnosis but was declined by the patient. After 1 year, he returned to our hospital with severe dyspnea caused by the enlarging tracheal tumors. A tracheal Ultraflex stent was inserted on an emergency basis and airway obstruction was relieved immediately. The bronchoscopic and panendoscopic biopsies were repeated, showing submucosal aggregation of oncocytic neoplastic cells in glandular arrangement with occasional colloid-like secretion microscopically (Figure 2, A). The tumor cells were immunoreactive

\footnotetext{
From the Division of Thoracic Surgery, Department of Surgery and Department of Pathology, ${ }^{\mathrm{b}}$ National Taiwan University Hospital and National Taiwan University College of Medicine, Taipei, Taiwan.

Received for publication Jan 15, 2007; revisions received Feb 17, 2007; accepted for publication Feb 23, 2007.

Address for reprints: Yung-Chie Lee, MD, PhD, Professor, Department of Surgery, National Taiwan University Hospital and National Taiwan University College of Medicine, No.7, Jhong-Shan S Rd, Taipei, Taiwan 100 (E-mail: wuj@ha.mc.ntu.edu.tw).

J Thorac Cardiovasc Surg 2007; 134:524-5

$0022-5223 / \$ 32.00$

Copyright $\odot 2007$ by The American Association for Thoracic Surgery doi:10.1016/j.jtcvs.2007.02.027
}

to cytokeratin, neuronspecific enolase, chromogranin (Figure 2,

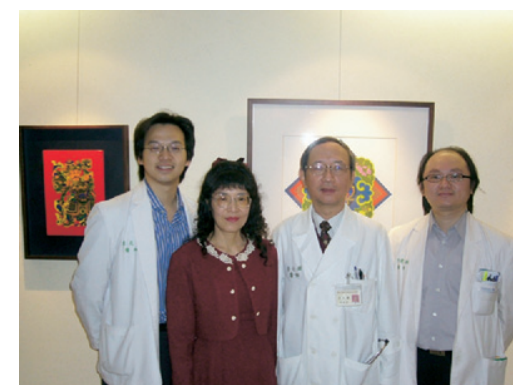

Drs Chen, Chang, Lee, and Pan (left to right) $B)$, and synaptophysin but negative for smooth muscle actin, indicative of a carcinoid. Diagnosis of atypical carcinoid tumor was reached owing to increased mitotic activity, nuclear polymorphism, increased cellularity with disorganized architecture, and focal tumor necrosis in places. Curative operation was precluded for advanced disease, and no effective chemotherapy was available for this rare neoplasm. Radiotherapy was unacceptable to the patient, and oral thalidomide was prescribed as a palliative therapy. During a 30-month follow-up, the patient is living well with the disease. Currently, the extent of disease remains stationary, with the patient reporting only mild dysphagia (Figure 1,B).

\section{Discussion}

Carcinoid tumors have been reported as occurring throughout the gastrointestinal tract, most commonly in the small bowel, appendix, and rectum, but they are exceedingly uncommon in the esophagus. They usually occur in older men who have dysphagia, demonstrating no clinical specificity compared with esophageal adenocarcinoma or squamous cell carcinoma. In all the reported cases, the patients had dysphagia, and only one had the carcinoid syndrome. $^{2}$ Therefore, it is difficult to differentiate esophageal carcinoid tumors clinically from adenocarcinoma and squamous cell carcinoma. The final diagnosis depends on the microscopic and immunohistochemical features. Because atypical carcinoid is an intermediate malignancy with aggressive ability, it is necessary to differentiate atypical carcinoid from other tumors. The histologic appearance of an atypical carcinoid is established according to the criteria proposed by Arrigoni, Woolner, and Bernatz ${ }^{3}$ : (1) increased mitotic activity in the presence of recognizable carcinoid pattern; (2) pleomorphism, nuclear irregularity, hyperchromatism, and abnormal nuclear/cytoplasmic ratio; (3) hypercellularity with disorganization of the architecture; and (4) areas of tumor necroses. Some authors suggest that the aggressive behavior of atypical carcinoid of the esophagus precludes the use of limited surgical resection and requires adjuvant chemoradiotherapy. ${ }^{2}$ In our case, the patient had advanced esophageal atypical carcinoid tumor with tracheal invasion, which required tracheal stenting. Oral thalidomide was prescribed because of our previous successful experiences with thalidomide as an adjuvant therapy for patients with malignant solitary fibrous tumor, margin-involved desmoids, and unresectable metastatic osteosarcoma. ${ }^{4}$ Thalidomide is postulated to have antiangiogenic activity through its ability to interfere with the vascular endothelial growth factor and basic fibroblast growth factor pathways. This activity was proved with apparent disease 

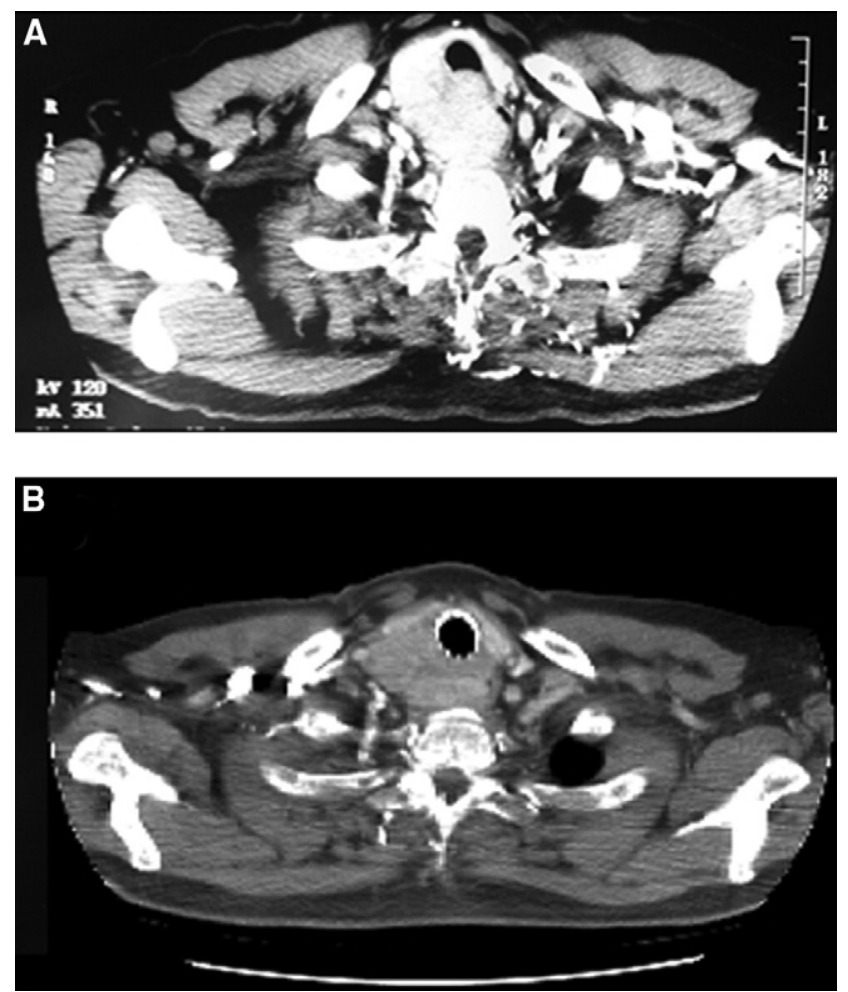

Figure 1. A, Initial chest computed tomographic scan showed the esophageal tumor with invasion to the trachea. B, Several months later, a follow-up scan showed the tumor size was stationary under thalidomide usage and the tracheal stent was noted in situ.

stabilization in a recent phase II study of patients with metastatic neuroendocrine tumors. ${ }^{5}$ After being followed up for 30 months, our patient still has a good quality of life except for only mild dysphagia.

In summary, esophageal atypical carcinoid is a rare disease. When it is localized, surgical intervention is the treatment of choice. However, when metastasis or local infiltration is present, the outlook is poor despite radical resection and adjuvant chemoradiotherapy. Our case demonstrates a worthwhile experience of palliative therapy with tracheal stent insertion and oral thalidomide for this advanced malignant tumor.

\section{References}

1. Tateishi R, Taniguchi H, Wada A, Horai T, Taniguchi K. Argyrophil cells and melanocytes in esophageal mucosa. Arch Pathol. 1974;98: 87-9.
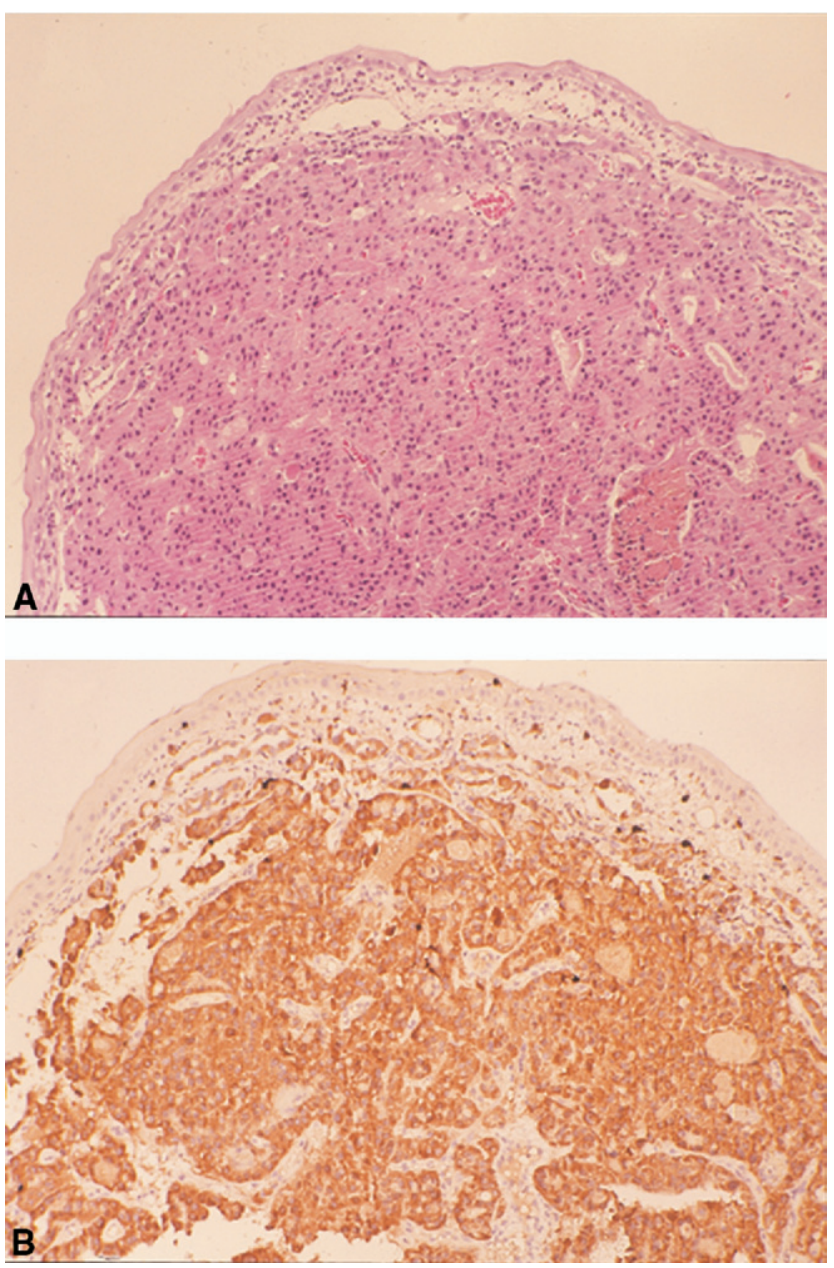

Figure 2. A, The polypoid nodule of the trachea demonstrated oncocytic neoplastic glands with occasional colloid-like secretion in the submucosa; B, Strongly chromogranin immunoreactive tumor cells.

2. Lindberg GM, Molberg KH, Vuitch MF, Albores-Saavedra J. Atypical carcinoid of the esophagus: a case report and review of the literature. Cancer. 1997;79:1476-81.

3. Arrigoni MG, Woolner LB, Bernatz PE. Atypical carcinoid tumors of the lung. J Thorac Cardiovasc Surg. 1972;64:413-21.

4. Tsai YC, Wu CT, Hong RL. Response of refractory osteosarcoma to thalidomide and celecoxib. Lancet Oncol. 2005;6:997-9.

5. Kulke MH, Stuart K, Enzinger PC, Ryan DP, Clark JW, Muzikansky A, et al. Phase II study of temozolomide and thalidomide in patients with metastatic neuroendocrine tumors. J Clin Oncol. 2006;24:401-6. 